\title{
Archéopages
}

Archéopages

Archéologie et société

40 | 04-07/2014

Villages

\section{La fouille de puits. Contraintes, protocoles et perspectives de recherche}

Christophe Tardy, Jean-Marc Fémenias, Christophe Pellecuer et Hervé Pomarèdes

\section{(2) OpenEdition}

12 Journals

Édition électronique

URL : https://journals.openedition.org/archeopages/647

DOI : 10.4000/archeopages. 647

ISSN : 2269-9872

Éditeur

INRAP - Institut national de recherches archéologiques préventives

Édition imprimée

Date de publication : 1 juin 2015

Pagination : 156-161

ISSN : 1622-8545

\section{Référence électronique}

Christophe Tardy, Jean-Marc Fémenias, Christophe Pellecuer et Hervé Pomarèdes, « La fouille de puits. Contraintes, protocoles et perspectives de recherche », Archéopages [En ligne], 40 | 04-07/2014 mis en ligne le 01 juillet 2016, consulté le 22 janvier 2022. URL : http://journals.openedition.org/ archeopages/647 ; DOI : https://doi.org/10.4000/archeopages.647 


\title{
La fouille de puits Contraintes, protocoles et perspectives de recherche
}

\author{
Christophe Tardy Inrap \\ Jean-Marc Fémenias Archéopuits \\ Christophe Pellecuer UMR 5140 «Archéologie des sociétés méditerranéennes" \\ Hervé Pomarèdes Inrap, UMR 5140 «Archéologie des sociétés méditerranéennes»
}

\section{6}

Avec la collaboration de

- Nathalie Chardenon Inrap, UMR 5140 «Archéologie des sociétés méditerranéennes»

- Isabel Figueiral Inrap, UMR 5554 «Institut des sciences de lévolution de Montpellier»

- Olivier Mignot ${ }^{\text {Inrap }}$

- Yoann Pascal ${ }^{\text {Inrap }}$ aubaine du fait de la diversité des matériels qui y sont piégés et des possibilités qui sont offertes de saisir des informations chrono-culturelles, paysagères et environnementales inattendues. Les diverses expériences de fouilles de puits, acquises sur des opérations programmées et préventives depuis une douzaine d'années, ont conduit à mettre au point, tester et améliorer un protocole spécifique. Présentées ici au travers de certains sites archéologiques languedociens, de nouvelles méthodes d'intervention permettent d'aborder les structures profondes de manière optimale, dans des contextes variés et qui dépassent certaines contraintes à la fois techniques, spatiales ou géologiques.

La réalisation, dans des conditions règlementaires, d'une fouille manuelle au sein d'un conduit de puits doit prendre en considération également un ensemble de mesures de prévention des risques, qu'ils soient mécaniques pour la manutention de charges ou électriques, en lien avec l'utilisation de matériels spécifiques (treuillage, éclairage, ventilation, pompage). Ces contingences nécessitent l'élaboration d'un protocole précis pour la conduite de l'opération. Une étude préalable de faisabilité de l'intervention sur un puits doit tenir compte de l'accessibilité de la structure, de la sécurité de l'installation de la plateforme et des moyens humains mis à disposition pour la mise en œuvre de la fouille.

En effet, depuis la fouille en profondeur, l'extraction et la remontée des matériaux et des mobiliers, jusqu’à leur traitement en surface règlementations strictes pour l'extraction des comblements. 
(évacuation, stockage, tamisage, échantillonnage, conditionnement), une série de plateaux techniques doivent être mis en place en accord avec l'ensemble du chantier de fouille. Les cheminements, la gestion des déblais et des circuits d'eau conditionnent le traitement des zones archéologiques alentour. Après vidange complète de la structure, l'étude de son parement et le relevé précis de son profil permettent d'apporter de précieuses informations sur sa construction et son mode d'alimentation en eau. Après la fouille, sauf contre-indication de la part de l'aménageur, il est préférable de reboucher rapidement le puits vidé, car la structure est possiblement fragilisée par le vide ainsi recréé.

L'investissement sur le terrain est donc important au cours de l'intervention, pour une durée moyenne de cinq à sept jours par puits, mais les gains scientifiques sont cependant substantiels et stratégiques dans l'interprétation générale du site.

\section{La nature des comblements}

Le comblement des puits varie d'une structure à l'autre, mais certaines convergences peuvent être observées. Nous présentons ici, à titre d'exemple théorique, un profil dessiné à partir du puits du site de Montferrier, établissement agricole du HautEmpire à Tourbes (Hérault), offrant la diversité des dépôts le plus fréquemment retrouvés [ill. 1]. Le fond du puits peut éventuellement révéler des dépôts interprétés comme «rituels» : certaines pièces de monnaie, des mobiliers très particuliers ou des restes de plantes symboliques. Les niveaux de fonctionnement, liés à l'utilisation du puits en tant que structure hydraulique et situés sous la nappe phréatique, sont particulièrement intéressants, car ils renferment, au-delà des artefacts, des restes organiques tombés accidentellement qui, sans ces conditions anaérobies, se seraient décomposés. En l'absence de curage, plusieurs couches de sédimentation peuvent s'accumuler et multiplient ainsi la richesse de l'information enfouie. Lors de l'abandon de l'utilisation du puits, la structure est condamnée. Le puits, dès lors, sert de dépotoir et l'intérieur est comblé, plus au moins rapidement, par toutes sortes de détritus produits à proximité. Les premiers éléments de mobilier rejetés sont en général ceux directement en lien avec la structure de puisage (crochet, seau, potence, margelle...) marquant du coup l'arrêt de l'accès à la ressource en eau. Le remplissage du conduit peut se faire avec de la simple terre, des détritus domestiques ou d'artisanat, des cadavres (d'animaux, plus rarement d'humains) et le plus souvent des matériaux de construction (pans entiers de maçonnerie, gravats, briques, tuiles...), etc. La préservation du matériel organique fragile dépend alors du niveau de la nappe phréatique encore active. Lors de la phase ultime de rebouchage, sous l'effet du tassement, il peut subsister un dernier creux dû à l'affaissement progressif du comblement. Ce bouchon supérieur, comblé tardivement, recueille bien souvent des mobiliers archéologiques correspondant à des occupations ultérieures. Le puits fonctionne ainsi comme collecteur d'écofacts extrêmement divers : résidus botaniques (pollens et spores, feuilles, graines, fruits, bois et charbons, algues...) et zoologiques (escargots, insectes, macrofaune, microfaune, ichtyofaune, coquillages...). Ils se retrouvent piégés dans les sédiments lors des phases successives de fonctionnement puis d'abandon de la structure. Ces dépôts s'accumulent de manière différente: de façon fortuite et naturelle (amenés par le vent, par le ruissellement ou par la gravité), ou, volontairement ou non, par le biais de multiples activités humaines et animales. Les puits sont des ensembles clos où se trouvent mêlées des informations de types variés qu'il faut décrypter, interpréter et retranscrire en termes de milieux environnants et de pratiques humaines (activités agricoles, pastorales, alimentation, utilisation de matériaux...) à replacer dans le contexte du site et de son évolution au cours de l'occupation. Des hypothèses interprétatives sont alors proposées afin de saisir la diversité de milieux autour du puits [ill. 2]. Les environnements s'organisent schématiquement en cercles concentriques depuis les bâtiments et les annexes vers les parcelles limitrophes et jusqu'aux paysages agraires ou « naturels» les plus éloignés.

\section{La gestion des mobiliers issus de milieux humides}

La conservation exceptionnelle de certains éléments est notamment constatée pour l'instrumentum et la vaisselle métallique. Il convient de mettre en place une prise en charge immédiate des objets, tant pour assurer une stabilisation appropriée que pour maîtriser quantitativement et qualitativement des séries et pour estimer et anticiper les interventions à venir. Des informations techniques précieuses, parfois éphémères (étamage, traitement de surface, couleur, aspect) doivent être observées et enregistrées, car les bains de stabilisation, de déchloruration et les traitements de restauration futurs peuvent parfois les amoindrir, les altérer, voire les masquer. Les étapes de traitement des mobiliers, adaptées à la nature des différents éléments (matériaux organiques, métaux, céramique, os), suivent des protocoles d'intervention précis, selon des précautions définies en amont, réajustées et susceptibles d'évoluer au besoin sur le terrain en fonction de la particularité des découvertes et des conseils dispensés par le laboratoire de restauration consulté. Une fois exhumés, tous les éléments (manufacturés ou non) sont rincés à l'eau courante afin d'ôter le sédiment enveloppant. Cette étape, sans incidence sur la préservation des mobiliers et la réalisation d'analyses éventuelles, permet de réaliser un premier tri, de premières identifications 


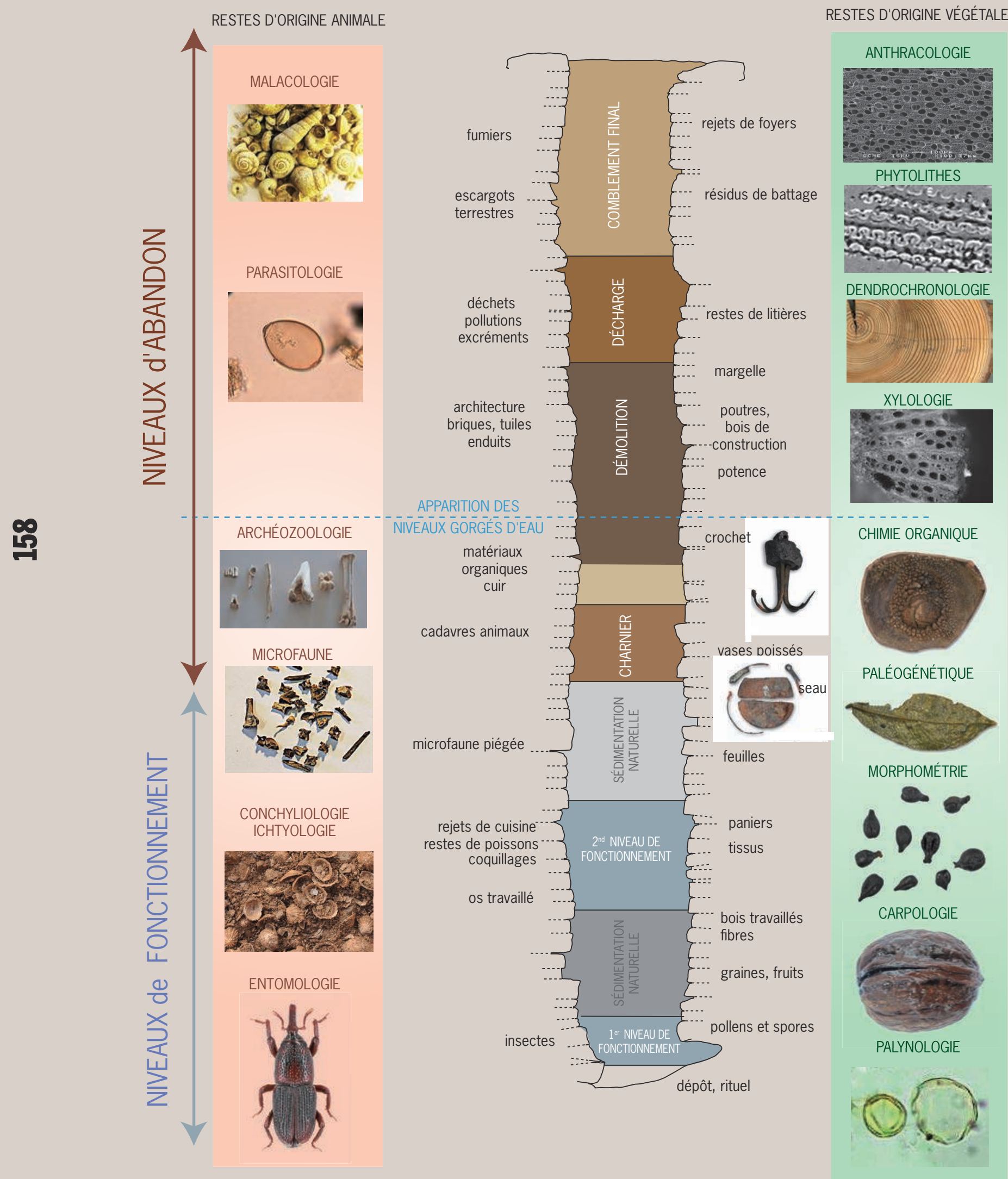

1. Profil type de puits présentant l'étendue des potentialités en matière d'études spécialisées à partir des nombreux mobiliers archéologiques et matériaux organiques conservés dans les comblements. 
nécessaires à la constitution d'inventaires exhaustifs et à la réalisation de leur couverture photographique et à celle d'un dossier d'archivage où est consigné l'état sanitaire des mobiliers au sortir du puits. Un espace de stockage est à prévoir sur le terrain, afin de permettre une gestion et un contrôle régulier des conditionnements différenciés des vestiges selon leur nature.

\section{Les perspectives de recherche}

L'équipe «Territoires et Sociétés de l'Antiquité et du Moyen Âge» du laboratoire «Archéologie des sociétés méditerranéennes ${ }^{\mathbf{1}}$ a accordé une place importante au thème de la fouille des puits et de l'exploitation des résultats obtenus dans ce type de contexte si particulier, dans son programme quadriennal 2011-2014. L'émergence de ce questionnement s'est imposée comme une évidence dans ce cadre collectif et pluriinstitutionnel. Des pratiques, de nouvelles expériences de terrain, une actualité de la recherche liée à des opérations préventives d'envergure, pouvaient être prises en compte pour alimenter des thèmes de réflexion qui fondent dans la durée les recherches de ce laboratoire. Ces échanges sont aussi facilités par une complémentarité entre l'expérimentation de la recherche programmée, qui en Languedoc a été menée pour ce type de fouille lors d'opérations dans les agglomérations protohistorique et galloromaine de Lattara et d'Ambrussum, et la généralisation de cette approche, un véritable changement d'échelle des pratiques grâce à l'archéologie préventive. Une table ronde tenue à Nîmes en 2011, à l'initiative de notre équipe et à l'invitation de la direction interrégionale Méditerranée, a permis un large tour d'horizon des interventions réalisées ces dernières années et une discussion avec les spécialistes engagés dans l'interprétation des résultats (Tardy et al., 2011). À partir de quelques exemples significatifs - bien d'autres aspects auraient pu être utilisés pour notre propos -, il est possible de montrer comment ces contextes, les découvertes récurrentes qui ont pu être faites dans ces milieux humides et les études qui ont pu être engagées, permettent des éclairages renouvelés, et même parfois une autre façon d'aborder certains thèmes.

Une meilleure appréciation de l'environnement technique des fermes et des villae méridionales est un de facteurs de connaissance de ces établissements ruraux que l'on envisage sans idée préconçue depuis que le moulin hydraulique, après les pressoirs, est devenu un équipement fréquemment attesté (Brun, Borréani, 1998). Les pièces de bois d'œuvres rejetées dans les puits permettent une réelle appréciation des capacités des charpentiers gallo-romains et de leur savoirfaire (Chabal, 2012). À partir de la multiplication de ces témoignages, les discussions sur la maîtrise de la ferme triangulée, de la pratique exceptionnelle à la diffusion la plus large, sont déterminantes pour la restitution des édifices dont la fouille ne révèle que des substructions. Les possibilités de portée, la diversité des essences utilisées ne sont plus envisagées depuis les prétendues limites qu'imposeraient les ressources de la forêt méditerranéenne. On peut attendre beaucoup des découvertes faites dans les puits pour mieux connaître des éléments de machinerie, treuils, godets de noria, voire de possibles systèmes de pompage (Bouet, 2005; Brun, Fiches, 2007).

Dans le domaine de la production viticole, les témoignages de tonneaux connus dans le Midi (Marlière, 2001, p. 197; Marlière, 2002) peuvent provenir de puits, mais ils sont encore trop rares pour remplir les chais vides de dolia que l'on a pu identifier ou pour affirmer la place du commerce du vin en vrac à côté des amphores. Leur existence même justifie cependant que l'on avance des modèles alternatifs de production selon les secteurs géographiques de la Narbonnaise et selon les périodes. Les quelques exemples connus appartiennent à la période du Haut-Empire, à l'époque où la fabrication des amphores semble prédominante. De même, on savait par les sources comme par des attestations archéologiques éparses que la poix était abondamment utilisée dans la production viticole, celle-ci parmi un panel très large d'autres activités consommatrices de ce distillat. La plupart des récipients retrouvés dans les puits en sont recouverts ou en contiennent, indiquant une utilisation quotidienne et un accès facile à cette matière première quasi stratégique, à l'égal d'autres ressources identifiées de longue date et dont le rôle déterminant dans l'économie gallo-romaine pourra ainsi être reconsidéré. Sa fabrication, bien identifiée dans les piémonts du Massif central, suggère des spécialisations pour certains secteurs des cités méridionales ou des provinces limitrophes pour alimenter les bassins d'activité de la plaine (Trintignac, 2003).

On s'interroge enfin sur la question de la typologie, du profil des établissements ruraux antiques, que l'on a abordée à partir des années 1980 grâce aux résultats foisonnants de la prospection pédestre dans une région peu favorable aux révélations aériennes. Les classifications automatiques élaborées dans le cadre des programmes Archaeomedes I et II (Favory, Van der Leeuw, 1998) ont permis de décrire ces sites en cinq classes, allant du simple bâtiment annexe, à vocation agraire, jusqu'à l'agglomération, selon des variables communes et de manière homogène. Depuis ces expériences, l'apport considérable de l'archéologie préventive, avec la multiplication des fouilles de fermes et de villae, vient renouveler nos approches et constitue un défi pour les chercheurs de nos équipes.

La construction d'une typologie, qui bénéficierait de cette grande diversité de plans inédits, ne peut constituer qu'une étape préliminaire dans la réflexion à engager. Ces bâtiments, ces équipements participent, selon les points d'observation de l'université de
l'mo associa

Montpellier, le CNRS et le ministère de la Culture et de la Communication, en partenariat avec l'Inrap.

\section{品}



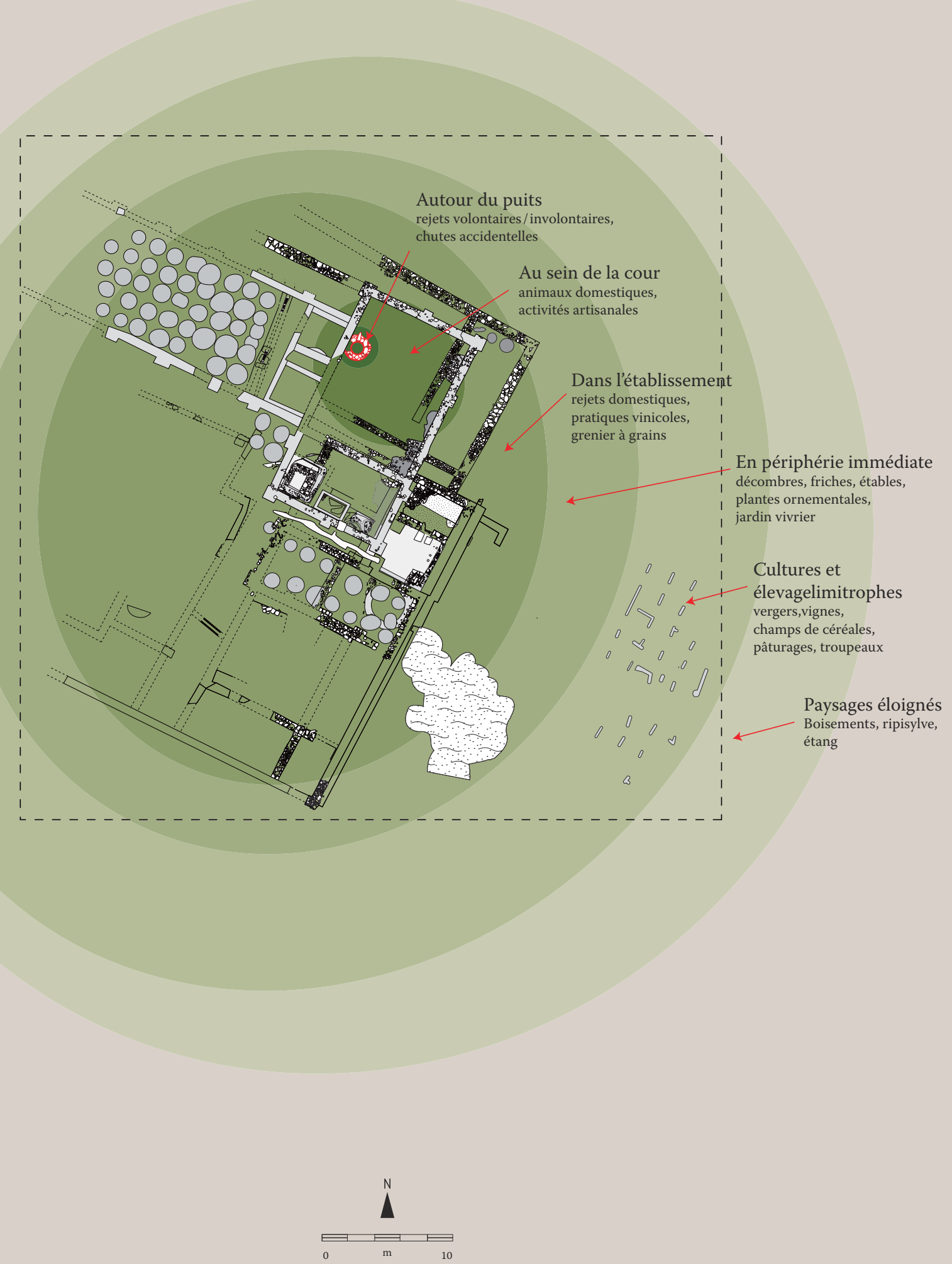
paysages ruraux, de systèmes de production ou, à une échelle supérieure, de systèmes agraires dont une meilleure connaissance permettra de mieux définir de futurs classements au-delà du simple aspect formel des constructions. La fouille des puits, dans les régions méditerranéennes, apporte le milieu humide inespéré qui manquait pour amplifier les études paléoenvironnementales. Lorsque l'on peut croiser données bioarchéologiques et résultats de grands décapages (par exemple, Figueiral et al., 2014), la viticulture, dont les traces sont omniprésentes, apparaît dominante du fait de son poids économique et de la localisation du vignoble à proximité de l'unité de production, mais elle ne masque pas d'autres formes de cultures, plus discrètes dans notre documentation traditionnelle (emblavures, cultures sarclées, jardins...) ou l'exploitation de milieux diversifiés (humides, forestiers...).

Si la fouille des remplissages des puits est souvent brève et réalisée généralement en fin d'opération, l'exploitation des données est, quant à elle, souvent planifiée sur le long terme compte tenu du nombre de spécialistes impliqués en postfouille. La restitution de leurs travaux s'organise assez naturellement en deux phases: une première expression des résultats par discipline, garante du travail de chacun, puis des réflexions croisées et coordonnées, confortant ou non les perceptions et ouvrant à une meilleure interprétation du site étudié. Les délais contractuels des opérations et la dotation de moyens, parfois très limités, pour chaque discipline, constituent cependant de réels obstacles. Ces contingences brident également les perspectives de recherches pluridisciplinaires élargies et mieux programmées. À l'expérience, on constate en effet que la fouille des puits génère des référentiels et des bases de données exceptionnels stimulant tant des études transversales aux sites que des synthèses et, par voie de conséquence, une valorisation scientifique et des publications de premier ordre.

Ces interrogations se sont imposées comme une évidence au regard de l'émulation scientifique et interinstitutionnelle qu'offre ce type d'investigations. Les objectifs sont de promouvoir le développement de fouilles de puits dans maints territoires et de garantir à de plus nombreuses occasions la collecte d'informations uniques et inédites. On l'aura compris, celles-ci sont en effet susceptibles d'éclairer d'une nouvelle manière le fonctionnement des sites et le comportement socioéconomique des communautés sur lesquels les recherches sont prescrites et les avancées scientifiques attendues.

Références bibliographiques

Bouet A. (DIR.), 2005, Aquam in altum exprimere. Les machines élévatrices d'eau dans l'Antiquité, Actes de la journée d'étude tenue à Bordeaux, 13 mars 2003, Bordeaux, Ausonius (coll. «Scripta antiqua », 12), $169 \mathrm{p}$.

BRUN J.-P., BorRÉANI M., 1998, « Deux moulins hydrauliques romains en Narbonnaise. Villae des Mesclans à la Crau et de Saint-Pierre/Les Laurons aux Arcs (Var)», Gallia, 55, p. 279-326.

BRUN J.-P., FiCHES J.-L. (ÉD.), 2007, Énergie hydraulique et machines élévatrices d'eau dans l'Antiquité, Actes du colloque international tenu à Vers-Pont-du-Gard, 20-22 septembre 2006, Naples, Centre J. Bérard (coll. du Centre J. Bérard, 27), $257 \mathrm{p}$.

Chabal L., 2012, « Poutres, appareillages et mobiliers en bois », in Fiches J.-L. (DIR.), Quatre puits de l'agglomération routière gallo-romaine d’Ambrussum (Villetelle, Hérault), Montpellier, Association de la Revue archéologique de Narbonnaise (coll. Suppl. à la $R A N, 42$ ), p. 65-132.

FAVORY F., VAN DER LEeUW S, 1998, «Archaeomedes, une recherche collective sur la dynamique spatiotemporelle de l'habitat antique dans la vallée du Rhône: bilan et perśpectives ", Revue archéologique de Narbonnaise, 31, p. 257-298.

Figueiral I., Pomarèdes H., Court-Picon M., Bouby L., Tardy C., Terral J.-F., 2014, «New insights into Mediterranean Gallo-Roman farming: a closer look at archaeological wells in Southern France. Archaeol Anthropol Sci », [en ligne] DOI 10.1007/s12520-014-0181-8

MARLIÈre E., 2001, «Le tonneau en Gaule romaine», Gallia, 58, p. 181-201.

MARLIÈre E., 2002, L'Outre et le tonneau dans l'Occident romain, Montagnac, éd. Monique Mergoil (coll. «Monographies Instrumentum», 22), $205 \mathrm{p}$.

Tardy C., Pellecuer C., Fémenias J.-M., 2011, «La fouille des puits, à propos d'expériences récentes dans le midi de la France, Nîmes », Bulletin de liaison, 21, p. 32-39.

TrintignAC A., 2003, «La production de poix dans la cité des Gabales (Lozère) à l'époque galloromaine», in LePETz S., MATTERNE V. (DIR.), Cultivateurs, éleveurs et artisans dans les campagnes de Gaule romaine. Matières premières et produits transformés, Actes du VI e colloque de l'association AGER, Compiègne, 5-7 juin 2002, in Revue archéologique de Picardie, 1/2, 12, p. 239-248.

Breuil J.-Y., Chardenon N., Barberan S., Bovagne M., Houıx B., à paraître, Un assemblage original de mobilier du Haut-Empire découvert dans un puits de la fouille du parking Jean-Jaurès à Nîmes (Gard) ?, Colloque international de Poitiers «Les mobiliers archéologiques dans leur contexte, de la Gaule à l'Orient méditerranéen ", 27-29 octobre 2014.

Fabre L., Figueiral I., Tardy C., Compan M., 2009, «Le puits de la villa de Montferrier à Tourbes (Hérault): des données paléoenvironnementale pour un essai de restitution d'une exploitation agricole antique», in Collectif, The Territory and its resources, Girona, Laboratori d'Arqueologia i prehistoria/Institut de Recerca Historica/Universitat de Girona/Grup de Recerca Arqueologica del Pla de l'Estany (coll. « Studies on the Rural World in the Roman Period», 4), p. 73-83.

FICHES J.-L. (DIR.), 2012, Quatre puits de l'agglomération routière gallo-romaine d’Ambrussum (Villetelle, Hérault), Montpellier, Association de la Revue archéologique de Narbonnaise (coll. Suppl. à la RAN, 42), 372 p. 